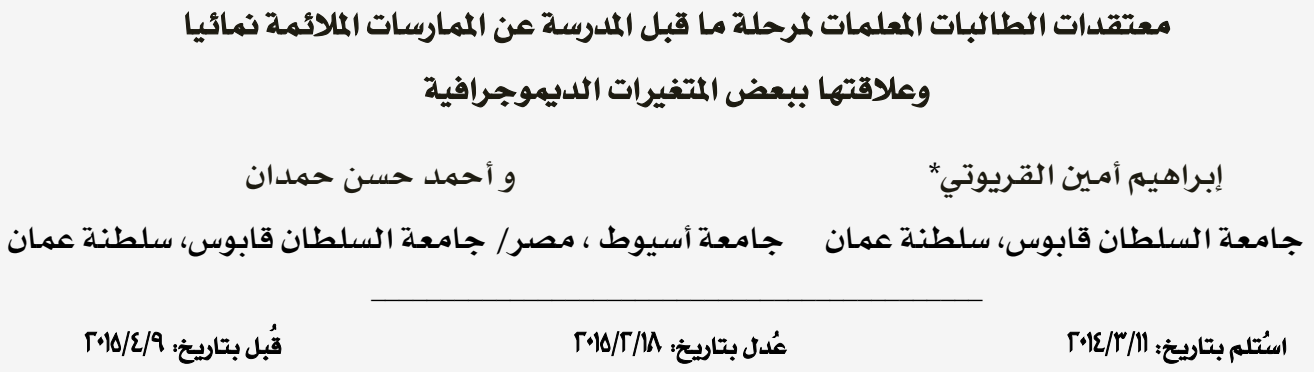

المستخلص: هدفت الدراسة الحالية إلى التحقق من معتقدات الطالبات المعلمات تخصص طفل ما قبل المدرسة نحو الممارسات المملائمة

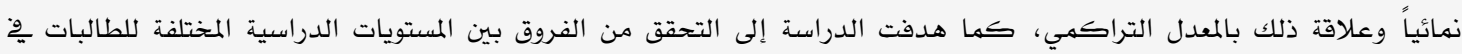

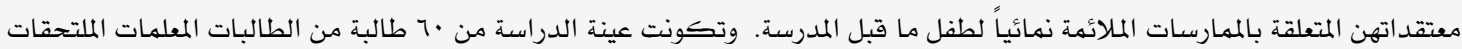
بيرنامج قسم طفل ما قبل المدرسة بكلية التربية وِّج جامعة السلطان قابوس من مستويات دراسية مختلفة (سنة أولى وثانية وثالثة ورابعة). استخدمت الدراسة مقياس معتقدات المعلم الذي يتكون من ثلاثة أبعاد هي المنهج المتكامل/المنهج الاجتماعي-الثقايو، المهارات الموجهة

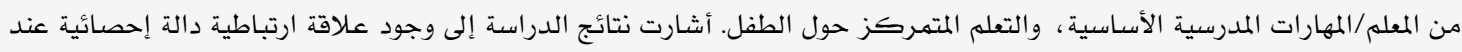

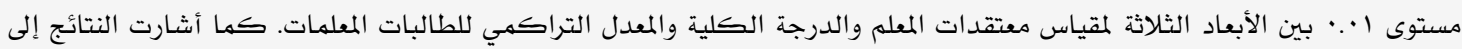

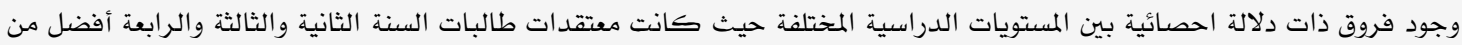

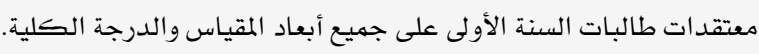
كلمات مفتاحية: المعتقدات، الطالبات المعلمات، الممارسات الملائمة نمائياً، مرحلة ما قبل المدرسة.

\title{
Student Teachers' Beliefs about Developmentally Appropriate Practices and Their Relationship with some Demographic Variables
}

\author{
Ibrahim A. Alqaryouti* \\ Sultan Qaboos University, Sultanate of Oman
}

\& Ahmed H. Hemdan

Assiut University, Egypt/ Sultan Qaboos

University, Sultanate of Oman

\begin{abstract}
The purpose of this study was to investigate female student teachers' beliefs towards developmentally appropriate practices (DAP) and the relationship between these beliefs and the GPA. It also aimed to investigate the differences in DAP beliefs among female student teachers at different years (1st through 4th year). Study sample consisted of 60 female students selected from the Early Child Education program at the College of Education, Sultan Qaboos University from different years (1st through 4th year). The study used the three subscales of teacher beliefs scale: (a) integrated/socialcultural curriculum, (b) teacher-directed/basic school skills and (c) child-centered learning. The results indicated a significant correlation at $\mathrm{p}=0.01$ among the three TBS subscales, the total score and GPA. Significant differences were also found between the different years where the beliefs of the second, third and fourth year students were better than first year students beliefs on all subscales and the total score.
\end{abstract}

Keywords: Beliefs. Female student teachers, developmentally appropriate practices, preschool children.

*ibrahimq@squ.edu.om 
كما تستند مبادئ المهمارسات المهلائمسة نمائياً

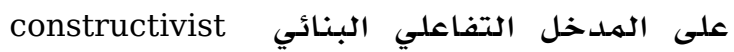
(Bredekamp \& Copple, 1997) interactive وقد أورد بياجيه أن الأطفال الصغار يقومون

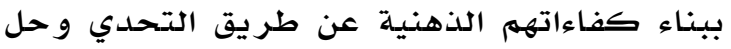

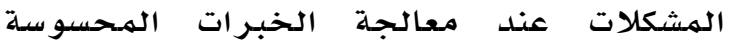
حيث ينهمك الأطفال (Stipek \& Byler, 1997) في تطوير فهمهم من خلال هذه الخبرات. وهم

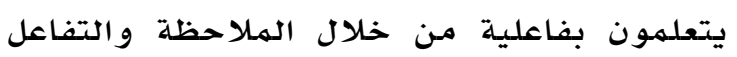

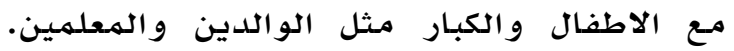
و علاوة على ذلك، فان اللعب هو الموقف الأول

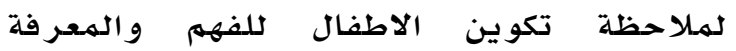
(Chaille \& Silvern, 1996). و وتقوم نظرية الاطفولة النهو

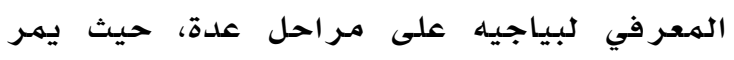

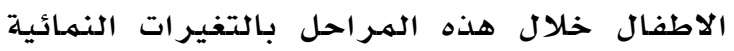

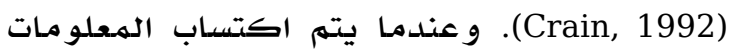

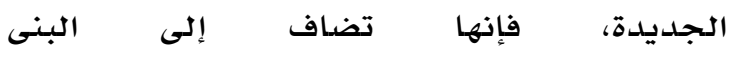
المعرفية schemas مـن خلال التمثيل، و الموائمهة،

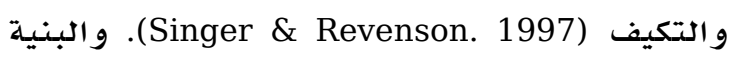

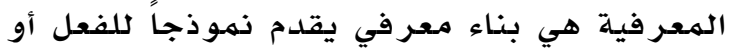

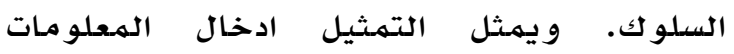

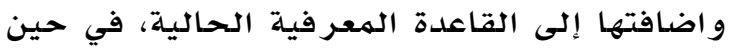

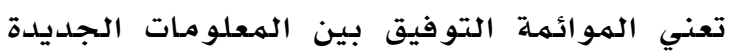

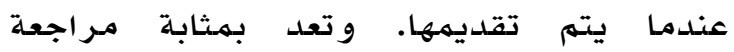
للهمعلومات، و يعد التكيف بمثابة تنظيهم المعلومات وتهات

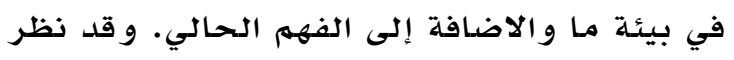
بياجيه إلى لعب الطفل على أنه وسيلة لزيادة النهو المعرفي، واعتقد أن الأطفال يسهمون بنشاط فعال في بناء معرفتهم وأنهم يتعلمون عن طريق العمل. (Singer \& Revenson, 1997) و أكد فايجوتسكي تأثير العوامل الاجتهـاعية و الثقافية على الهفاهيم التي نتعلهمها وكيفي

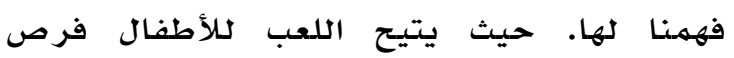

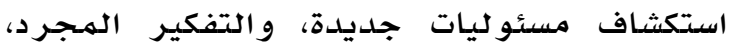

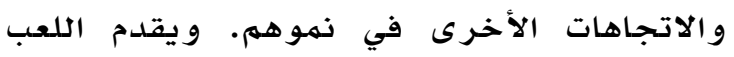

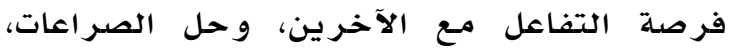

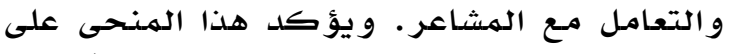

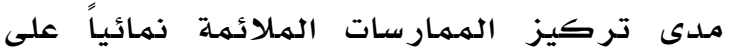

الممار سات الملائمة نمائياً Developmentally :Appropriate Practices (DAP) لاقت الهمـارسات الملائمهة نهائياً اهتماماً كبيراً

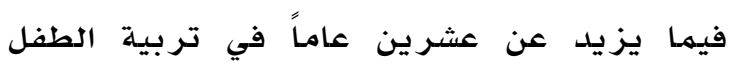

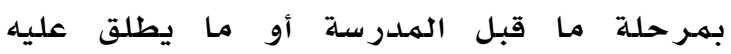

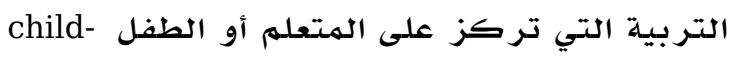
كرد فعل or learner-centered education للسيطرة المتزايدة للمهمارسات التي تركز فقط

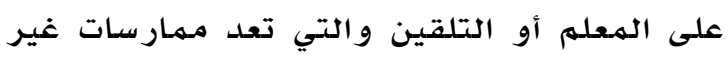

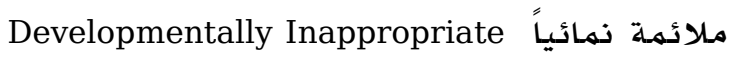
(Charlesworth, Hart, Burts, Practices (DIP) و وكان الدافع (Mosley, and Fleege, 1993) الرئيس لهذا الجهد هو الحاجة إلى التخلص من

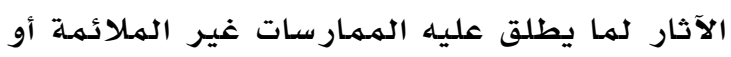
تقليدية. (McMullen, 1999)

و تعرف الممارسات الملائممة نمائياً على أنها الهمرفة الهتعلقة بكيفية نهو و وتعلهم الأطفال

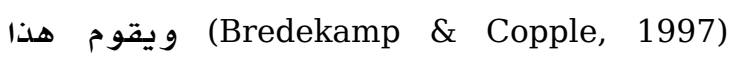
المدخل على قدرة المعلمين على فهم النمو الفردي لكل طفل وتخطيط خبرات التعلهم التي تلدعم هذا النمو بناء على الاحتياجات الفردية آدية.

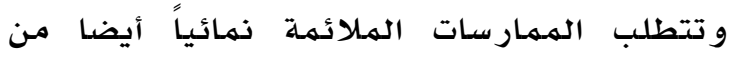

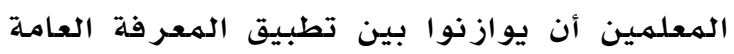

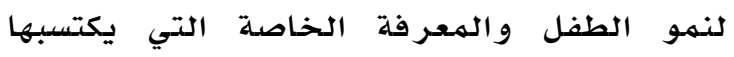
المعلم عن طريق تأسيس علاقة بين كل طفل

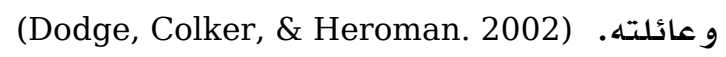

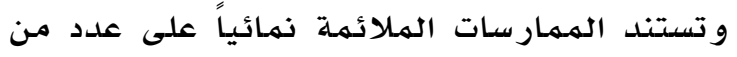

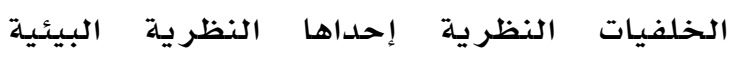
Bronfenbrenner's ecological model

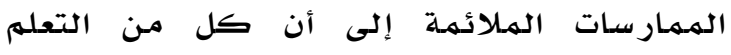

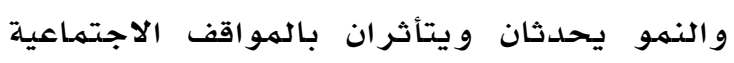
و الثقافية (Bredekamp \& Copple, 1997). و يسهم النهوذج البيئى في الهمارسات المـلائمة نمائياً في الهساعدة على فهم التأثيرات الثقافية الثياتئي

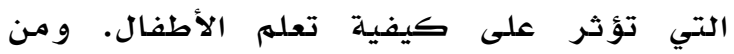
المفيد أن ننظر إلى نمو الطفل في سياق

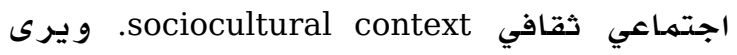

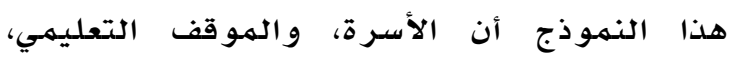
و الهجتهمع، والبيئة لها تأثيرات على نهو الأطفال. 
و مهمارسات معلمهي ما قبل الهمدرسلة في الولايات الهتحدة. و على الر غم من أن مقياسي معتقدات

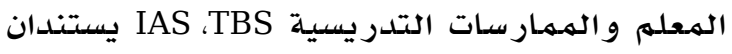

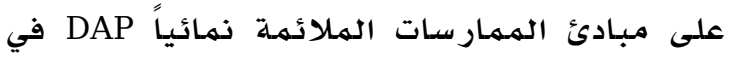
الو لايات المتحدة و الذي يعكس النظريات الغهيات الغربية في نمو الطفل، فهي لا تزال مفيدة في الدراسات

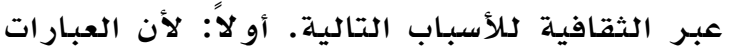
في المقياسين تغطي العديد من الجوانب الهامـة

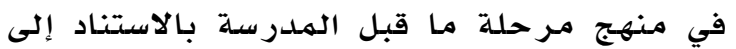
الهفاهيم العامة للمنهج بـاضلاضافة إلى الأبعاد

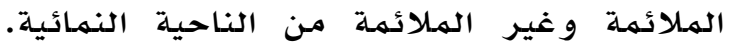

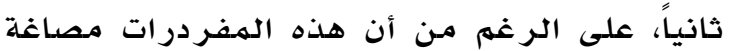

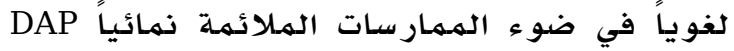

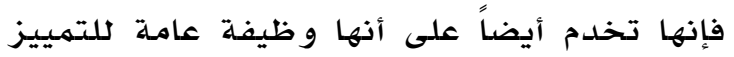
بين معتقدات المعلمين حيث يرون أنها ترتبط لهـا بمدى كبير من الهبيادئ و النظريات لنسمو وتعلهم

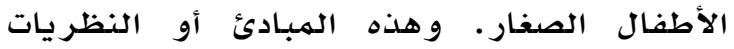
الغربية لها قيمـة ويتقبلها الباحثون و الههتمون في مجال طفل ما قبل المدررسـة عبر الثقافات على الرغم من التفضيلات أو أوجه التر أركيز

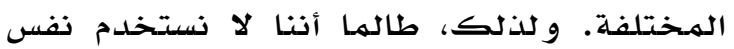
المفهوم من الممار سات الملائمسة نمائياً

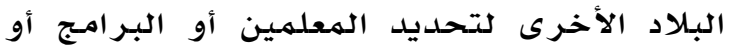

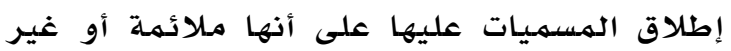

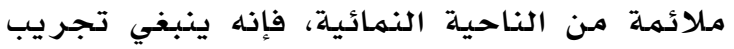

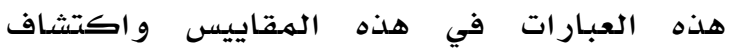

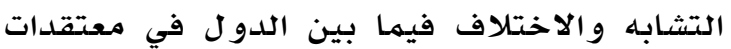
المعلمين المـرتبطة بتكامل الهـهـج، تسهيل النهمو الاجتماعي والانفعالي، تقديم الأنشطة اليدوية، و تقديم فرص اللعب في الهنهج.

و لعقود عديدة، اهتمت البحوث بعمليات التفكير للمعلمين و المعتقدات التعليمية و المعر فية بمثابة

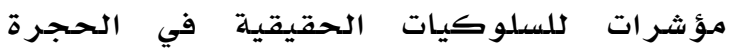

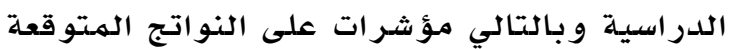

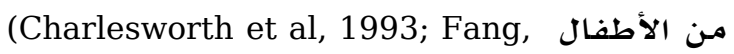
(1996; Pajares, 1992) و على الرغم من ذلك، فإن هناك عدد متزايد من الدراسـات البحثية التي

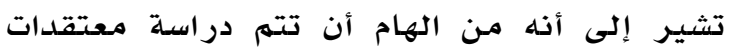

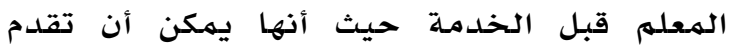

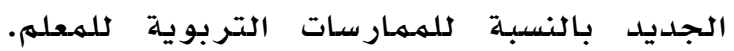

اللعب و السهاح للأطفال لاكتساب ومهمارسة هذه الههارات عن طريق التفاعل و التعلهم من الاخرين.

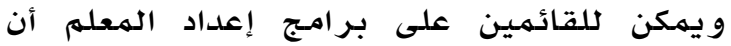
يسهموا في نمو معتقدات ومهمارسات المعلمين

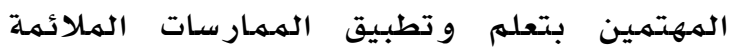
Robinson \& Hart, (Haupt, Larsen, نهائياً .1995)

\section{المعتقدات تجاه الممارسات الملائمة نمائياً:} منذ الثمانينات، استحوذ الاهتمام على دراسـة

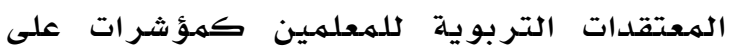
سلوكات المعلمين الحقيقية في الحجرة الصفية

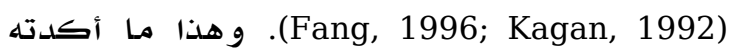
أغلب نتائج البحوث، ولذا فإن معتقدات الهعلم

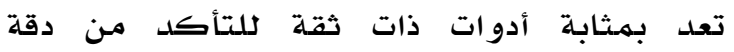

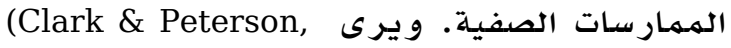

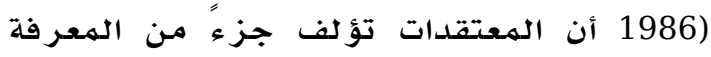
العامة للهعلمين التي من خلالها يتهكن المعلهم من إدراك و معالجة و فهم المعلومات في الحجرة الدراسية وذلك من أجل اتخاذ القرار وار مات. ووهناك

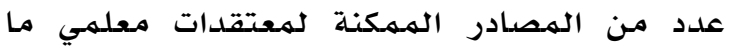
قبل المدرسة تشهل الخبرات الشخصية السابقة،

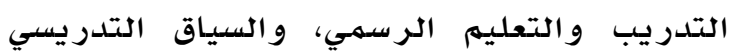

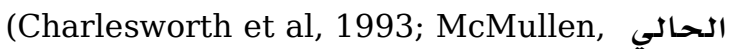
(1997

و أدت البحوث الحديثة في الولايات المتحدة

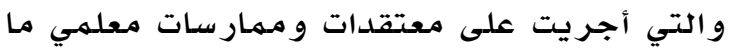

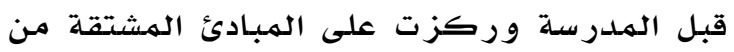

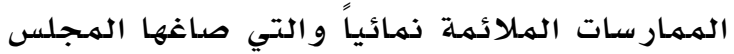

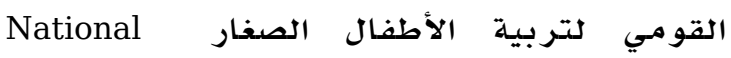
Association of Education for Young

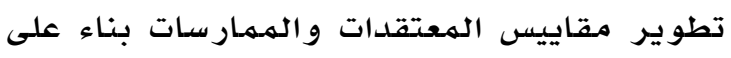

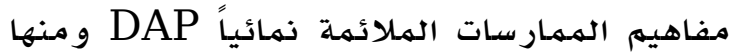
على سبيل المثال Charlesworth et al, 1991 and ،1993) Teacher Beliefs Scale (TBS) Instructional Activities Scale (IAS) استخدام هذه الهقاييس لدراسـة المعتقدات

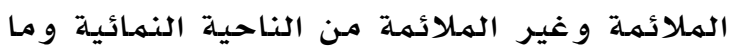

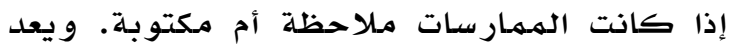
هاذان المقياسان من أشهر المقاييس الكميلة

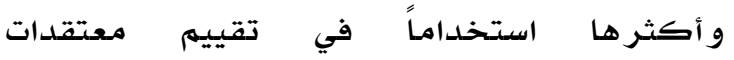


حديثة اهتهماماً متزايداً في معتقدات معلهي ما فيل

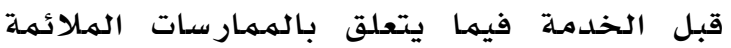
نمائياً في دول عديلدة حول لحه العالم.

يتضح من العرض السابق مدى أهمية دراسلة

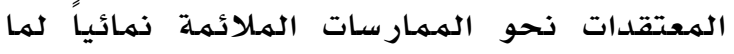
لها من أهمية كبيرة في بيان مدى لهو إتقانهن لههنة المستقبل. و على الرغم من تبني كلية المهية

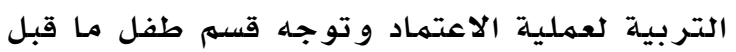
المدرسة نحو تبني معايير NAEYC و التي ترتبط

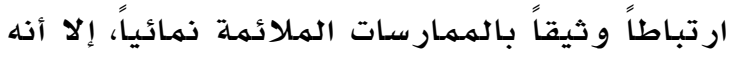

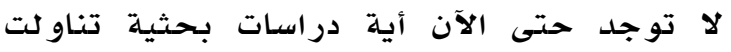
مدى إلمام الطالبات الهعلمات بتلك الهـ المهمارسات،

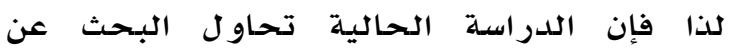

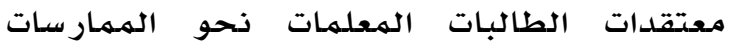
الملائمة نمائياً و التعر ف على مدى إلمامهن بها. أجرى Erdiller Akin (2013) دراسـة هدفت إلى معاتى فهم معتقدات معلمات ما قبل الخدمهة في مـرحلة

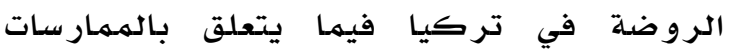

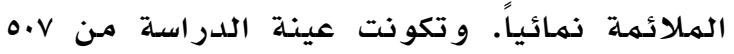

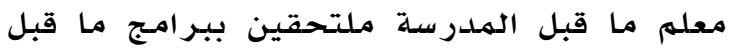

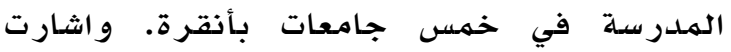
نتائج الدراسلة إلى أن معتقدات معلدمات ما قبل قبل

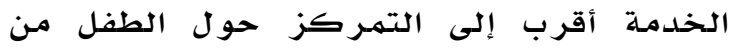

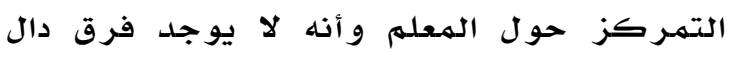

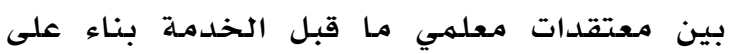
الخصائص و السمات الشخصية و التربوية. (2012a) Sakellariou \& Rentzou كما أجرى

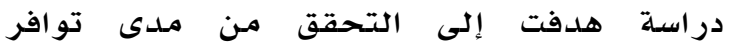

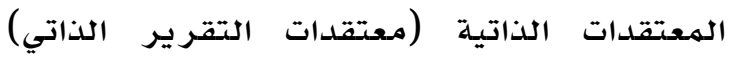
والمهمارسات الذاتية المـرتبطة بالمهمارسات

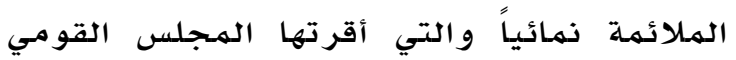

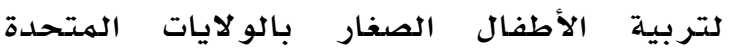
NAEYC

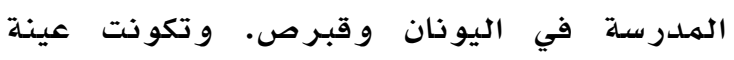

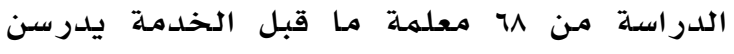
مقرر عن الطفولة الهبكرة في جامعة قبر صية مدية

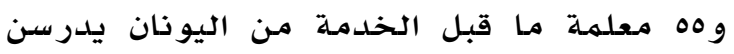

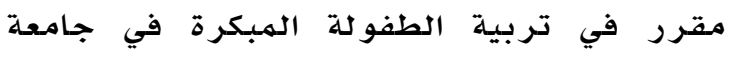

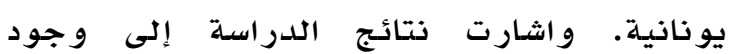

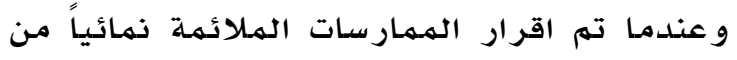
جانب المجلس القومي لتربية الأطفال الصغار NAEYC

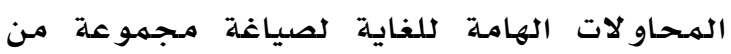

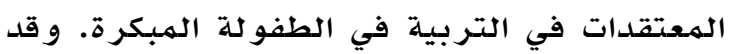

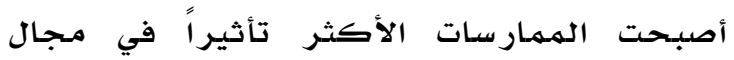

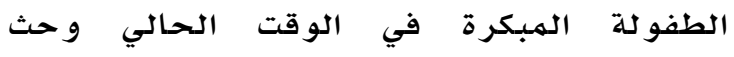

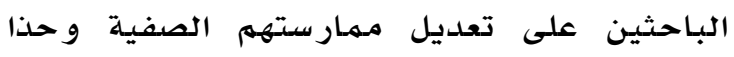

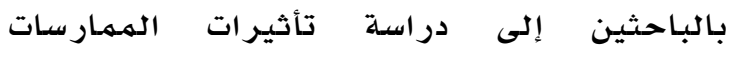
المهلائمة وغير الملائمة نمائياً على نمو الأطفال

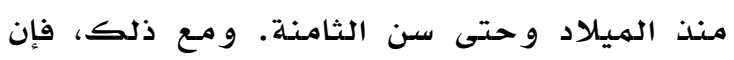
البحوث تشير إلى أنه غير شائع مثل الطرق التي

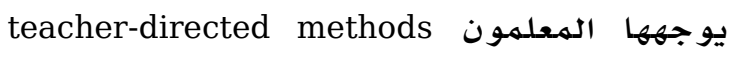
في أماكن ر رعاية الطفولة المبكرة. و ويبدو أن

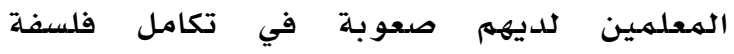

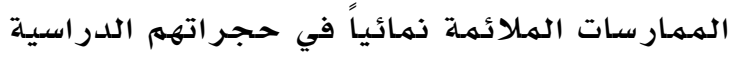
(Charleswoth et al, 1993) غالباً ما يمهارسون مـا يظنون أنه معتقداتهم عندما

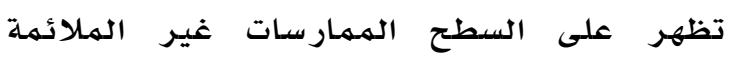

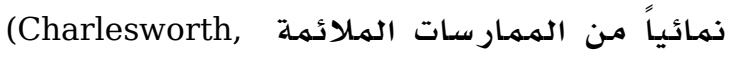

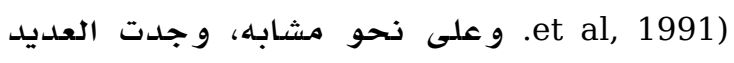

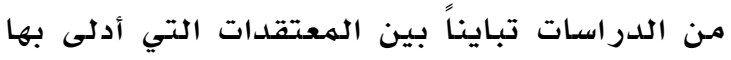

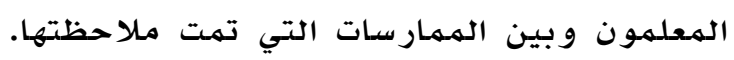
و عندما يكون هناك تباين، فإنه يكون غالباً في اتجاه تقرير المعلم لتطبيق متعمق للمهمارسات الملائمة نمائياً أكثر مهما ينعكس في مسمار سـاتهم

(Abbott-Shim, Lambert, \& McCarty, 2003)

و مـع ذلك، وومـع عدم ووفرة البحوث الهتعلقة

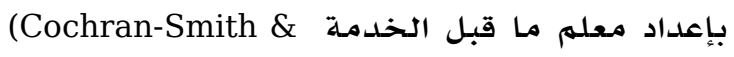
Zeichner, 2005) أجريت على معتقدات معلمي ما قبل الخدمـة فيما

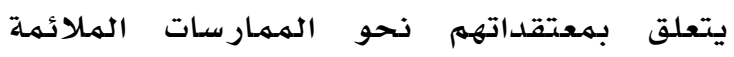

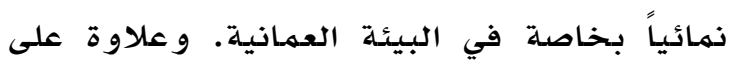

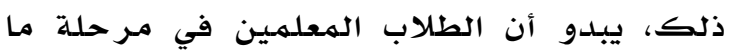

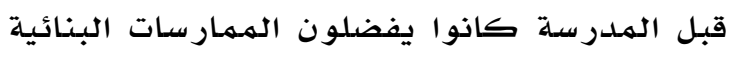

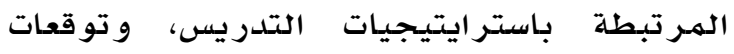

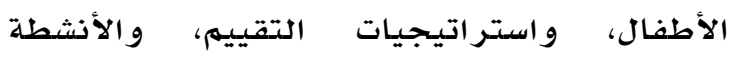

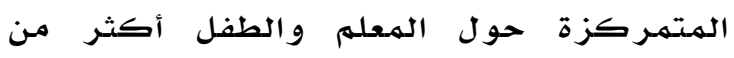

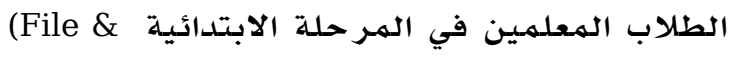
Gullo, 2002; 
الدراسية. و تشير نتائج الدراسـة إلى معتقدات الطلاب تتنباً بمهمار سـاتهم.

و هدفت دراسلة Sakellariou \& Rentzou (2011b)

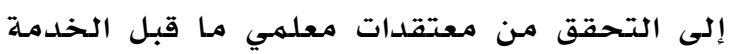

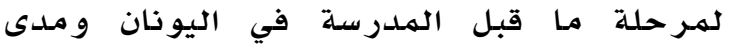
ارتباط تلك الهعتقدات بالمهمارسات الصفية.

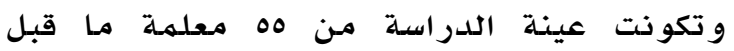
الخدمة يدرسن مقرر لطفل مـا قبل المددرسة في

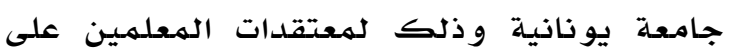
مهمارسـاتهم ومستوى معتقدات معلمي مـا قبل فيل الملدرسـة فيما يتعلق بالمـمارسـات الملائمسة نمائياً. وأستخدمت الدراسلة مقياس معتقدات المعلهم و ومقياس (Teacher Beliefs Scale. TBS) الهمـارسات التدريسية التهات

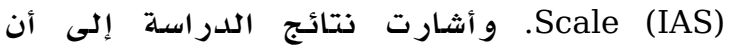

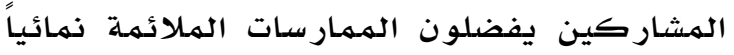

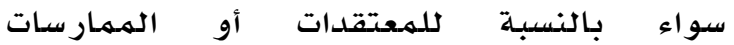

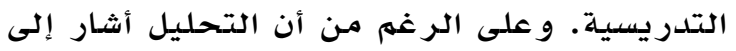

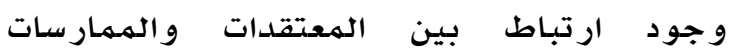

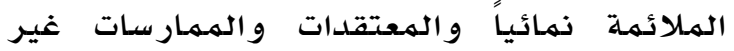

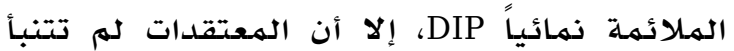
بالمهمار سـات.

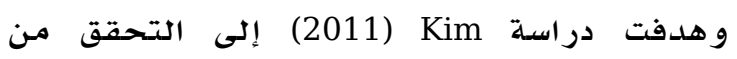

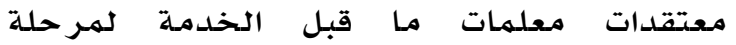
الروضدة فيما يتعلق بالمهمارسات الملائممة نمائياً

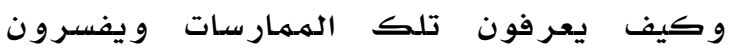
الهبادئ و خصائص الهمارسات. وتظهر الدراسة

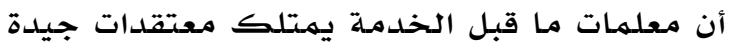
عن تلك المهمارسات.وتكونت عينـة الدراسلة مـن 70 المدرسة يدرسن ببر نامـج طفل ما قبل المدررسة

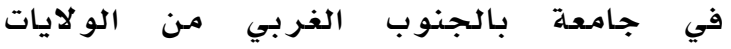

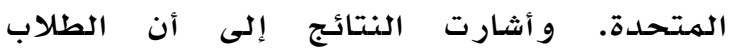

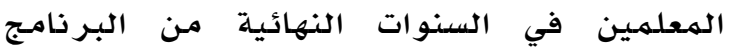
لديهم معتقدات أفضل مهـن في السنوات الأولى. كما أشارت النتائج إلى أن الهعلمين الذين كانت

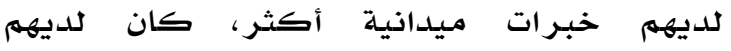
معتقدات أفضل.
اختالافات بين عينتي البحث في كل من اليونان و قبر ص. و قد وجد أن هناك علاقية بين البحسن الجنسية و متتوسط الدرجة الكلية في المقياس. و وعلاوة

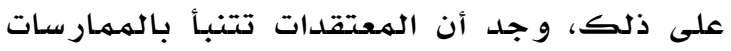
التي أقرت بها معلمات مـا قبل الخدمدة.

كما أجرى (2012b) Sakellariou \& Rentzou

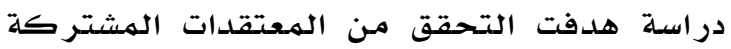
بين معلمي ما قبل الخدمـة لرياض الأطفال في

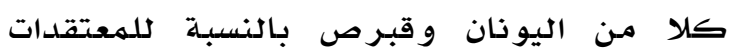
الذاتية و النوايا الذاتية المتعلقة بأهمية التفاعلات بين المعلم و الطفل و العلاقة بينهما. و وتكونت

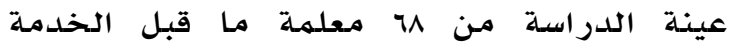
يلدرسن مقرر عن الطفولة الهبكرة في جامعة

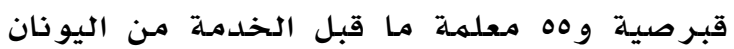
يدرسن مقرر في تربية الطفولة المبكرة في مديك

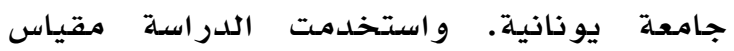
النوايا-الهمتقدات وهو مقياس مصهمم لقياس التفاعلات بين الطفل والهعلهم. وتمه استخدام معاملات الارتباط لبيرسون وتحليل التباين الأحادي في المقارنة بين متوسطات الدرجات

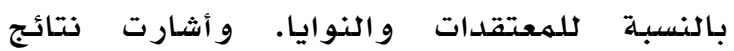
الدراسلة إلى أن الجنسية لا لت تلتبأ بمتوسط

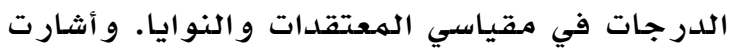
النتائج إلى أن معتقدات المعلمين تنبأت بنواياهمر.

وهدفت در اسـة Sakellariou) \& Rentzou, 2011a) إلى التحقق من المعتقدات والمهمارسات الذاتية لهعلمـات ما قبل الخدمة لهمرحلة رياض الأطفال

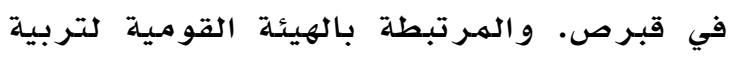

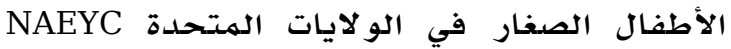

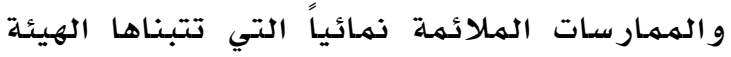
DAP

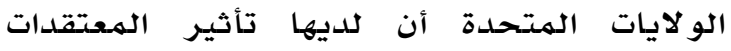

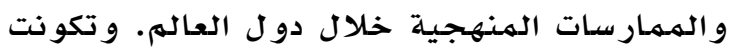

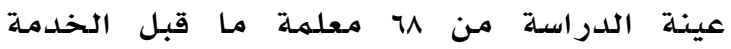
يدرسن مقرر لطفل ما قبل المدرسة في جامعة مهلة قبرصية. و أثـارت نتائج الدراسلة إلى أن الطلاب

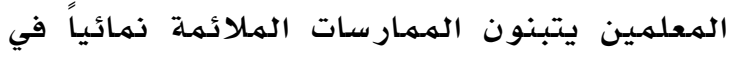

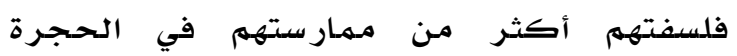


السنوات الأخيرة من الدراسة في أن الطلاب

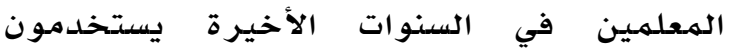

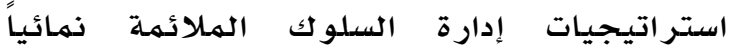
بصورة أقل. - الم كما هدفت دراسة (1997) Smith إلى التحقق من معتقدات الطلاب المعلمين المتعلقة بالمهمارسات

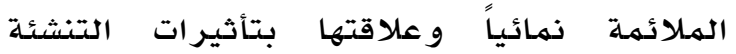

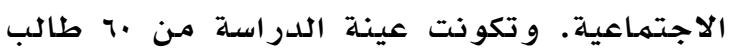

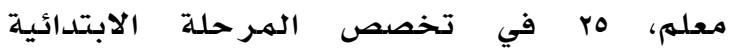
بالاضافة إلى تخصص الطفولة المبكرة،

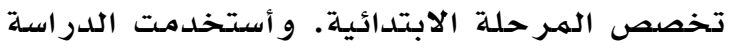

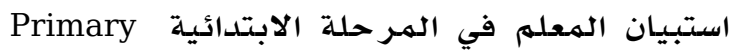
Teacher Questionnaire الداخلي. وكشفت نتائج الدراسلة أن الطلاب

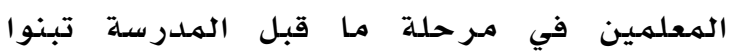

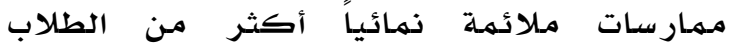

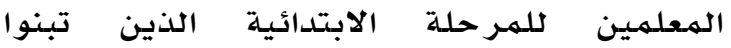
ممارسـات تقليدية بصورة أكبر .

دراسة هارون ووشاح (r.a.a) هدفت إلى استقصاء

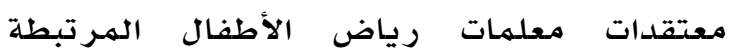
بالمهمارسة الملائمهة نهمائياً و المهمارسة وسلة التقليدية.

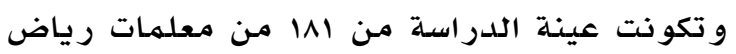

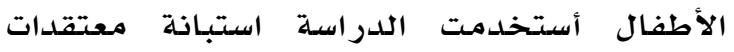

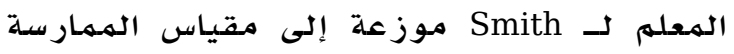

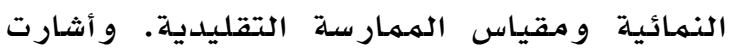
نتائج الدراسة إلى أن معلمات رئهيات رياض الأطفال

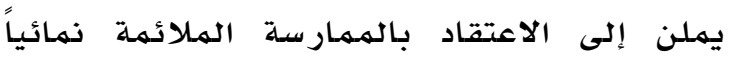

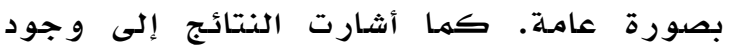
فروق ذات دلالة احصائية في معتقدات معلمات

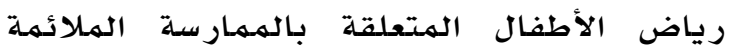
نمائياً تعزى للهمؤهل العلمي و ولصالح الهؤهل الأعلى.

يتضـح من العرض السابق ندرة الدراسات التي

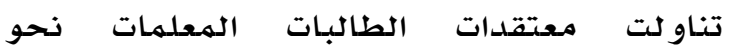

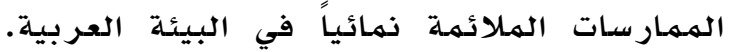
ويمكن استخلاص بعض النقاط منها: ا- أشارت معظم الدراسات إلى تبني الطالبات

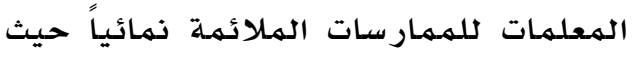
Erdiller Akin أشارت دريات
و هدفت دراسـة Jambunathan \& Counselman (2001) إلى التحقق من العلاقة بين المهمارسات

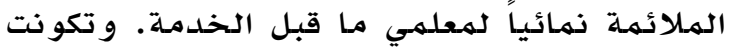

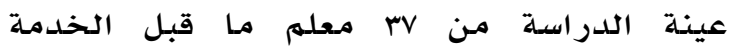

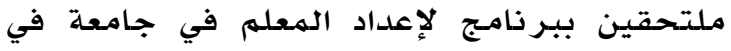

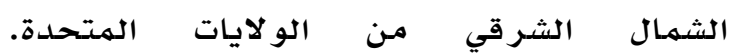
و أستخدمت الدراسة مقياس معتقدات و مهمارسات Teacher Beliefs and Practices Survey: المعلهم Preschool Inclusive Settings الدراسلة إلى أن معتقدات معلهي ما قبل الخدمة

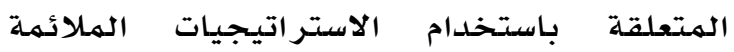
للتدريس ترتبط بصورة دالة بمهمارساتهم. و ومـع ذلك، لهم تكن معتقداتهم عن تطوير لهورئ المنهج،

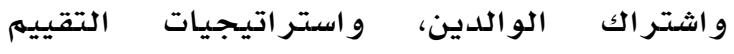
منعكسة بالضرورة في ممارساتهم الصفية. وهدفت دراسة File \& Gullo (2002) إلى التحقق

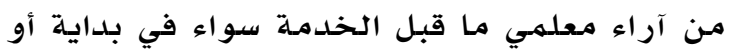

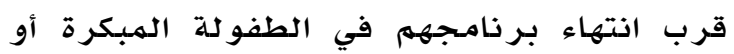

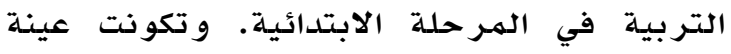

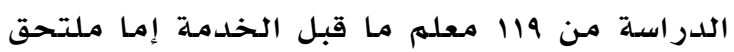
في برنامج لطفل ما قبل المدررسة أو بر نامـج

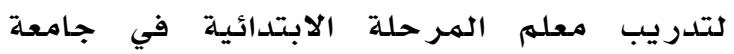

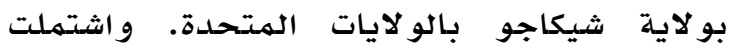

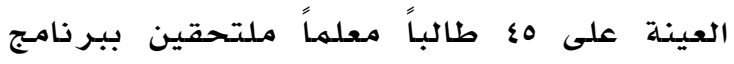

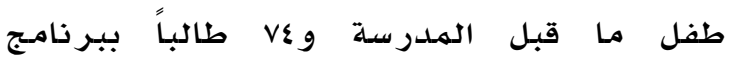

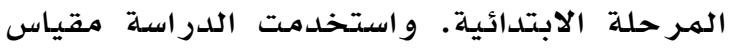

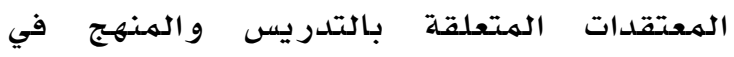
الصفوف الابتدائية وهو مقياس معدل مـن مقياس

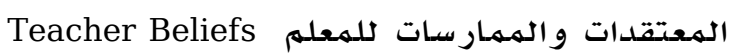
and Practices Survey إلى أن الطلاب المعلمين لبرنامـج طفل ما قبل

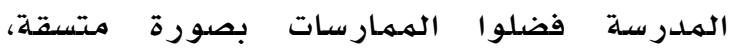
بالهقارنة مـع نظرائهم من الهمرحلة الابتدائية،

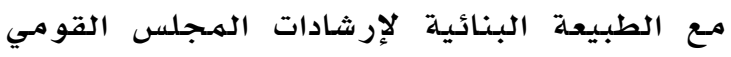
لتربية الأطفال الصغار NAEYC في مجالات عدة

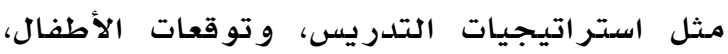

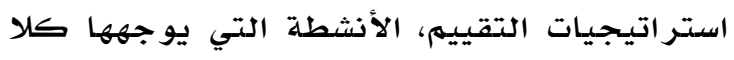

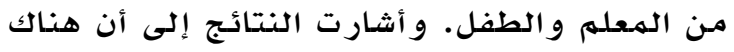
فرق دال بين الطلاب المعلمين في السنوات

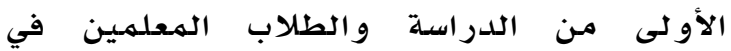


r. هل توجد فروق في معتقدات الطالبـات

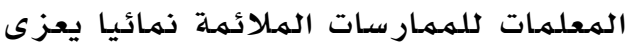

$$
\text { للمعدل التر اكمي؟ لمعئ }
$$

r. هل توجد فروق في معتقدات الطالبات

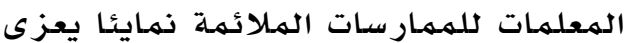

$$
\text { للهستوى الدر اسي؟ }
$$

أهمية الدراسة

ا. تنبع اهمية الدراسة النظرية من أهمية

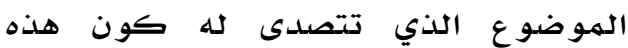
الدراسـة هي الأو لى من نوعها على حد علدم

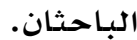

r. تتركز أهمية الدراسة التطبيقية في الاستفادة من النتائج في توجيه أعضيه أعضاء هيئة التدريس و والمسئولين في وزئ وزارة التربية والتعليهم عن الهمارسـات المـلائمهة نمائياً التي ينبغي وأن تحتئي المعلمات حتى يكتسبن المعرفة و الخبرة الكافية التي تؤهلهن للتعامل مـع الأطفال

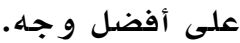
r. معظم بر امـج طفل ما قبل المدرسـة في

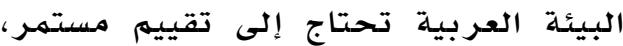
وهذا مـا يساعد أصحاب القرار في الوقوف

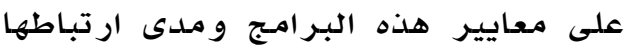
بالمعايير الدولية. ويدعم هذا سعي كلية

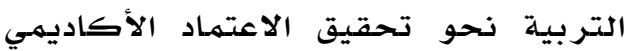

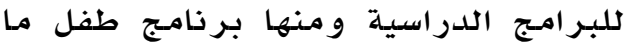

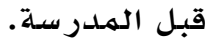
ع. تقدم نتائج الدراسة تغذية راجعة لمعدي برنامه طفل ما قبل الهـدرسة في الجامعة،

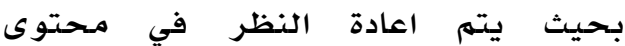

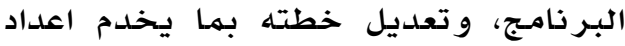

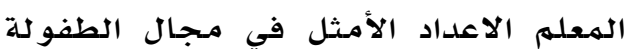
الهبكرة المبكر

\section{أهداف الدراسة}

1. التعرف على معتقدات المعلمهات للمهمارسات

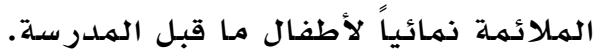

إلى أن بعد التمركز حول الطفل كان

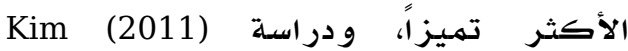
بالنسبة لامتلاك الطالبات لمعتقدات جيدة

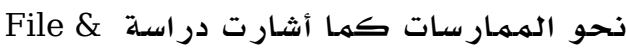
و Gullo (2002)

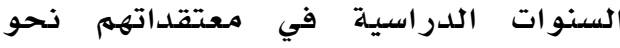
المهمارسات الملائمهة نمائياً.

r- تؤكد الدراسات على أهمية إمتلاك الطالبات المعلمـات للمهمارسات المهلائمة المهية نمائياً لما له من أهمية قصدوى في تشكيل مهار ساتهم التدريسية في البيئة الصفية. r- استخدام معظم الدراسات أشهر المقاييس والذي تم تطويره بناء على معايير NAEYC مرحلة ما قبل المدرسلة وهو مقياس Teacher Beliefs Inventory

مشكلة الدراسة

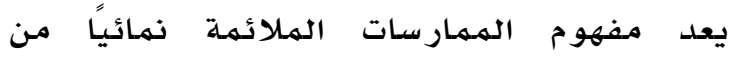
الهفاهيم الههمـة في برامج رياض الأطفال، لها له من انعكاسات ايجابية على تعلهم الطفل و تطوره في جميع جوانب نموه. و إيمان العاملين في رياض الاطفال بهذه الهمارسات وراعتادهادهم بأهميتها يعد الخطوة الاولى في تقديهم برامـج

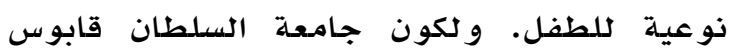
الجامعة الحكومية الوحيدة التي تطرح بر نامـج

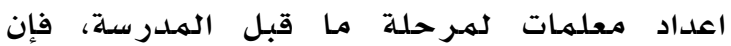
دراسـة مدىى اعتقاد المعلمات للمهمارسات الملائممة

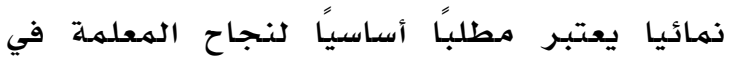

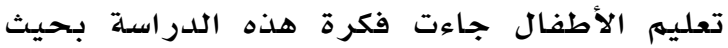

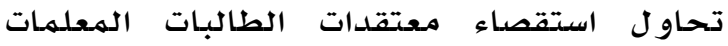

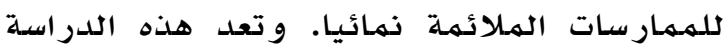
الأو لى التي تناقث موضوع المهمارسات الملائمة نمائياً في السلطنة على حلد على البه الباحثين.

أسئلة الدراسة

1. ما مستوى معتقدات الطالبات الهعلمات

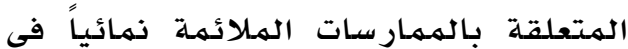
مرحلة ما قبل المـدرسة 
يقوم بتقدير الأهمية النسبية للهعتقد باستخدام

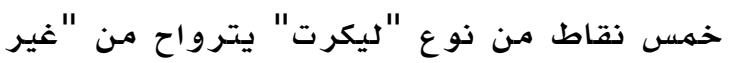

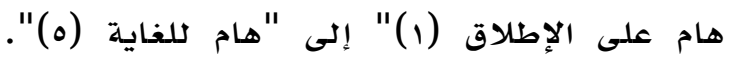

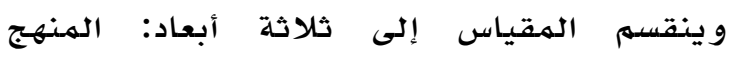

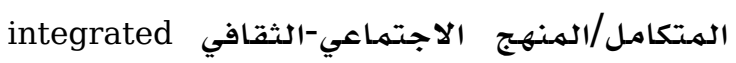

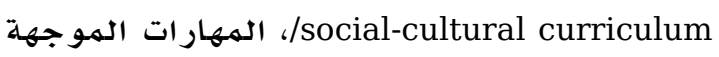
teacher- من المعلم/المهارات المـدرسية الأساسية الهوارات الهواتية من directed/basic school skills child-centered المتمركز .learning

وبالنسبة لثبات الاختبار، فقد تمتعت الهقاييس الفرعية الثلاثة والدرجة الكلية للمقياس باتساق

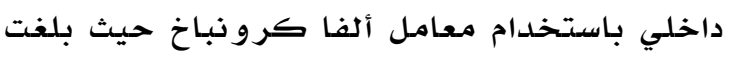

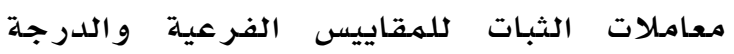

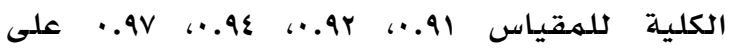

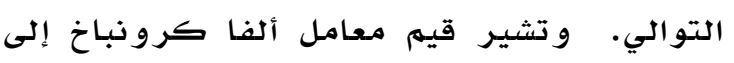
تهتع المقاييس الفرعية والدرجة الكلية بثبات

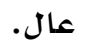

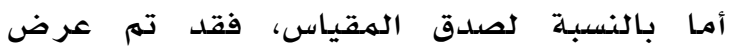
الهقياس بعد ترجمته على مجموعة مـ من الخبراء والهختصين في مجال طفل ما قبل المدررسة و علم النفس بجامعة السلطان قابوس. و أجمـع

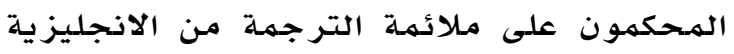

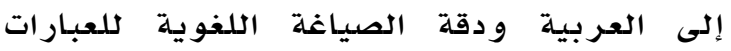

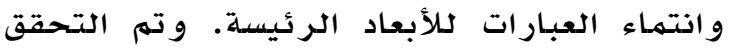

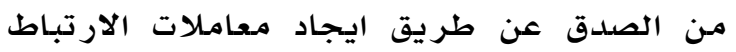
بين العبارة والبعد وبين البعد والدين الدرجة البهاد الكلية.

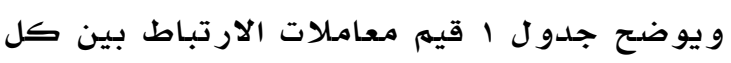

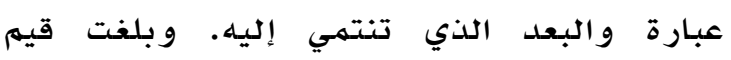

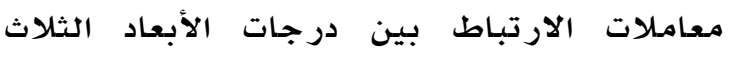

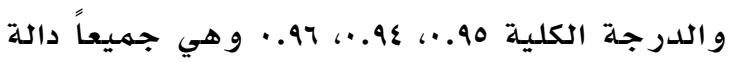
عند ا....

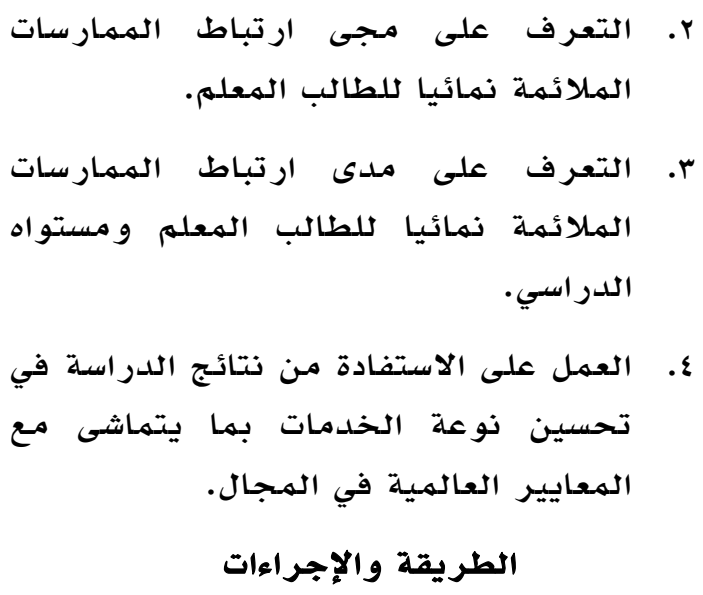

منهج الدراسة

استخدم البـاحثان المنهج الوصفي التحليلي،

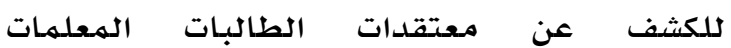
لمعتقداتهن حول اللهمارسات الملائمسة نمائيا، لدى الدى عينة من طالبات قسهم طفل مـا قبل المدررسة بجامعة السلطان قابوس. مجتمـع الدراسة وعينتها

تألف مجتهـع الدراسلة من جميع الطالبات

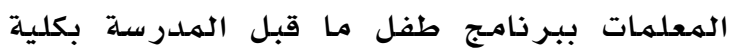

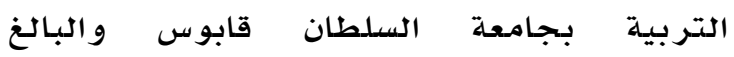

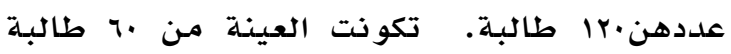

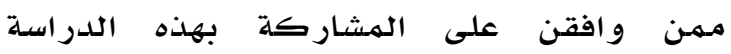
والاستجابة إلى أداتها من المستونيات الدرواسية الأربعة. أداة الدراسـة

تم تجميـع البيانات في هذه الدراسـة باستخدام مقياس معتقدات المعلم Teacher Beliefs Scale

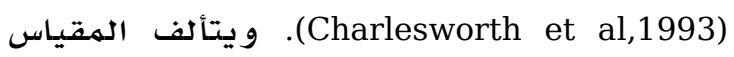
من بـ عبارة تقيس المعتقدات الملائمـة وغير

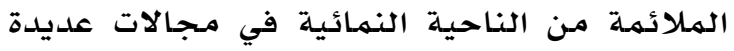

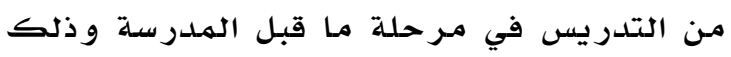
بالاستناد على إرشادات المجلس القومس مي لتربية

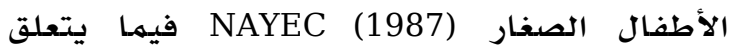

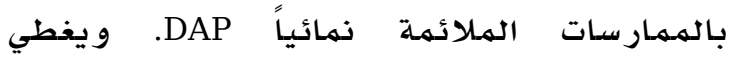
الهقياس معظم جوانب منهج الطفولة المبكرة

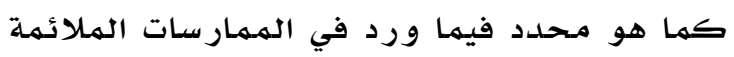
(Bredekamp \& Copple, 1997) عبارة في المقياس معتقداً ويطلب من الفرد أن 
من مساعدي البحث في توزيع الاستبـانات

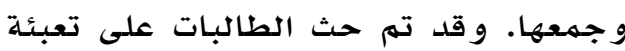
الاستبانة بصورة تطوعية وتم إخبار هن أن وهن

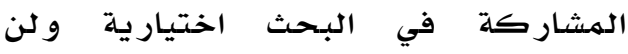

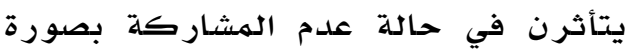

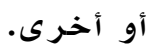

r. وبعد جمـع الاستبانات، تم احصاؤها،

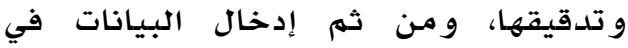

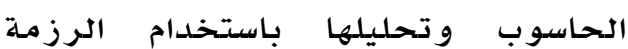

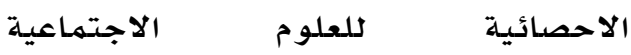

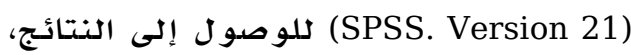
وون ثم تحليلها و مناقشتها.
إجر اءات الدراسة

اتبع الباحثان مجموعة مـن الاجر اءات البحثية

تمثثلت فيما يلي:

ا. إعداد الأداة و التأكد من صدقها وثباتها.

r. تحديد مجتمـع الدراسة، ثم اختيار عينتها

و ذلك من خلال إحصاء عدد الطالبات

الملتحقات في بردامج قسهم طفل مـا قبل

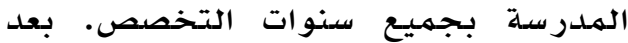

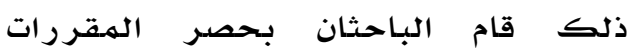
الدراسية التي يدرسن فيها الطلبات خلال

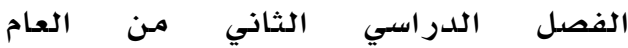

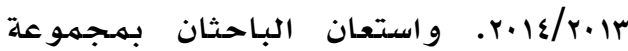

جدول 1

قيم معاملات الارتباط بين عبارات مقياس معتقدات التعلم

\begin{tabular}{|c|c|c|c|c|c|}
\hline البعد الثالث & العبارة & البعد الثاني & العبارة & البعد الأول & العبارة \\
\hline 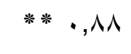 & r & *** & 1 & *** & $\varepsilon$ \\
\hline$* *, 9$ & $\wedge$ & $* * ., 09$ & $r$ & ** & 。 \\
\hline$* *, 9$ & 9 & $* * ., 00$ & 7 & $* *, \wedge 9$ & TE \\
\hline$* *, \wedge 9$ & 11 & *** & v & ** $\cdot$, & ro \\
\hline$* * \cdot, \wedge \mathrm{V}$ & ir & *** $\cdot, \wedge \varepsilon$ & 1. & $* *$ * & TV \\
\hline *** & $r$. & $* * *, 09$ & ir & 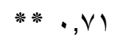 & rA \\
\hline$* *, 79$ & ry & $* * \cdot \wedge 1$ & $1 \leq$ & $* *, V \nearrow$ & Mr \\
\hline 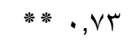 & rq & *** $\cdot, 7$ & 10 & " & זr \\
\hline$* *, 9$ & $r$. & $* * *, \wedge Y$ & 17 & 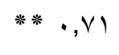 & $r \varepsilon$ \\
\hline$* * ., 01$ & 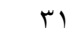 & $* * \cdot, \wedge 1$ & IV & $* * \cdot, \wedge 9$ & ro \\
\hline & & $* * *, Y V$ & 11 & $* * \cdot, \wedge \Lambda$ & Tr \\
\hline & & *** $\cdot, 07$ & 19 & & \\
\hline & & ד & rI & & \\
\hline & & $* * \pi$ & rr & & \\
\hline & & $* * ., 0 \wedge$ & rT & & \\
\hline
\end{tabular}


تقييم في برنامج الروضدة أمراً"، و الفقرة ج

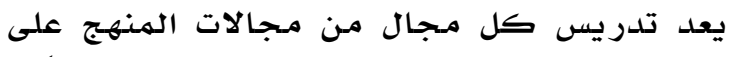
أنه موضوعات منفصلة في أوقات منفصلة أمراً. للإجابة عن السؤال الثاني الذي نص على "هل

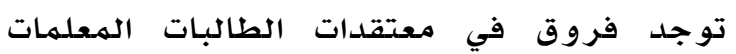

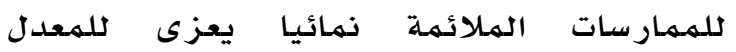

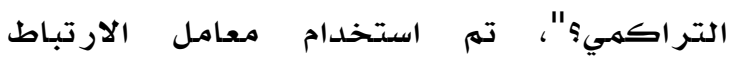

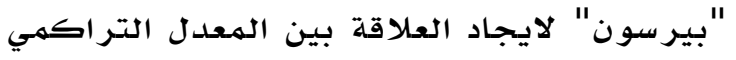
الذي تم إدخاله حسب نظام التقديرات المعمول به في الجامعة على ع نقاط. المقاييس الفرعية

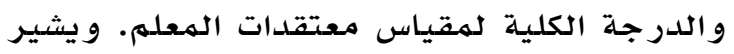
جدول r إلى وجود علاقة ارتباطية متوسطة دالة

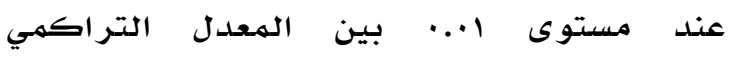
للطالبات والمقاييس الفرعية و الدرجة الكلية الكية للمقياس.

\section{النتصائج}

للإجابة عن السؤال الأول الذي نص على "مـا

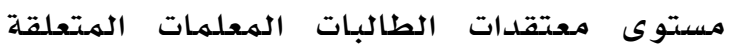

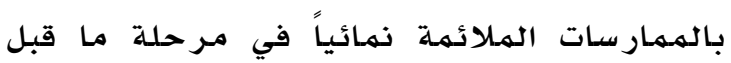

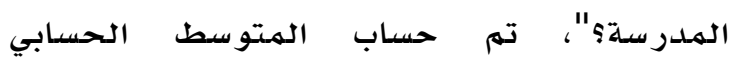

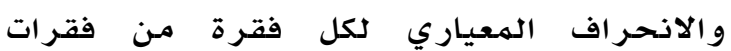

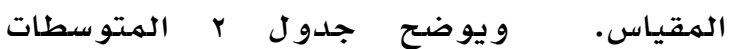
و الانحر افا ت المعيارية لفقرات ات مقياس معتقدات

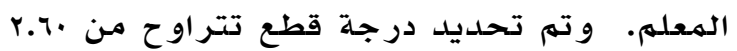

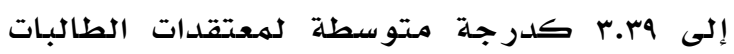

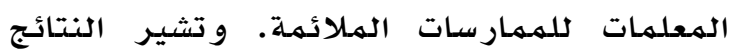
إلى أن مستوى المعتقدات لدى الطالبات يفوق

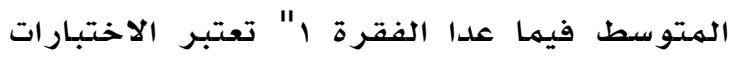

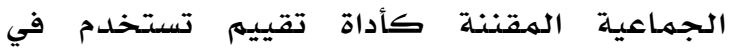

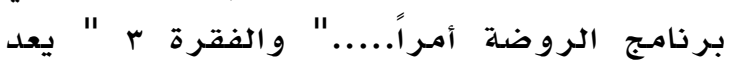

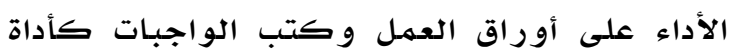

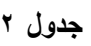

المتوسطات الحسابية والانحرافات المعيارية لفقرات مقياس معتقات المعلم

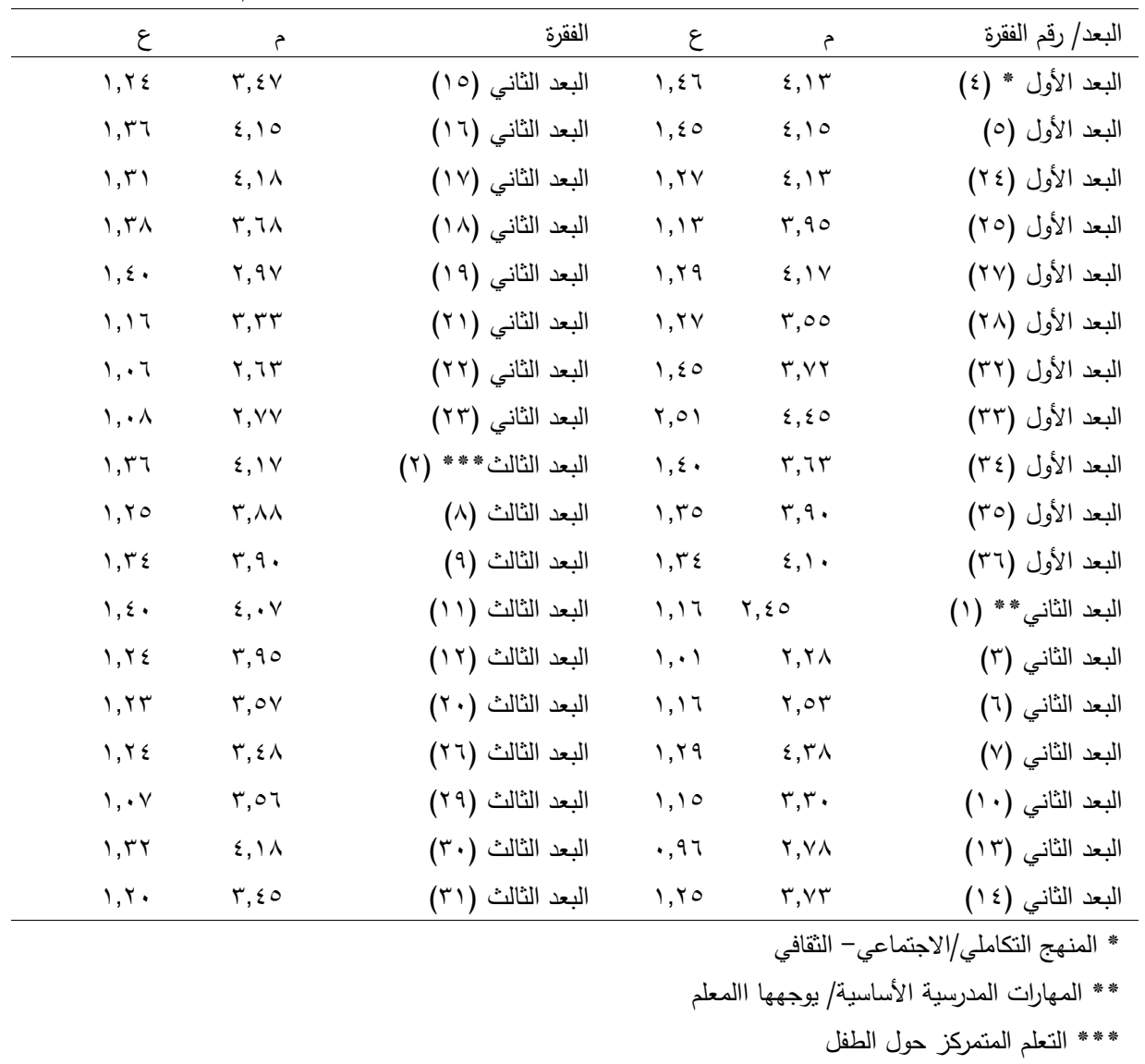


جدول ratris

معاملات الارتباط بين المقاييس الفرعية لمقياس معتقدات المعلم واللارجة الكلية والمعدل التراكمي

\begin{tabular}{|c|c|c|c|c|}
\hline الدرجة الكلية & البعد الثالث & البعد الثاني & البعد الأول & \\
\hline$* *, 09$ & $\% *$. O V & ** & $* * .09$ & المعدل التراكمي \\
\hline$* *, 97$ & $* *, 94$ & $* \cdot, \wedge \leq$ & & البعد الأول \\
\hline * *, $9 \leq$ & $* * *, \wedge \Sigma 1$ & & & البعد الثاني \\
\hline$* * .97$ & & & & البعد الثالث \\
\hline
\end{tabular}

البعد الأول: المنهج التكاملي/الاجتماعي-التقافي

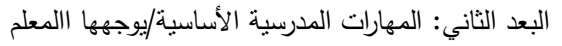

البعد الثالث: التعلم المتمركز حول الطفل

بين السنـة الأولى و الر ابعة لصالتح الر ابعة. و بلغت قيهمة حجم الأثر •r, •، سا, •، 19, • على الترتيب.

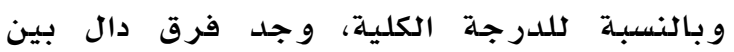
السنـة الأولى والثانية لصدالح الثانية، ووجلد فرق وجله دال بين السنـة الأولى والثالثة لصالح الثالثة، ووجد فرق دال بين السنـة الأولى والى ابعة الفئ

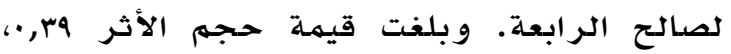

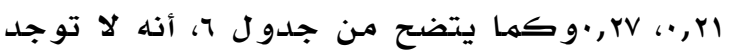
فروق دالة بين السنوات الثانية والثالثة، و الثانية

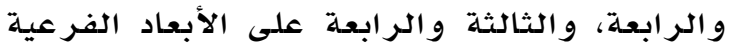

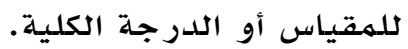
جدول ؛

يوضح متوسطات الرتب للمقاييس الفرعية والدرجة الكلية لمقياس معتقدات المعلم

\begin{tabular}{|c|c|c|c|}
\hline متوسطات & العدد & السنة & \\
\hline الرتب & & الدراسية & \\
\hline $1 \wedge, \cdot 7$ & 11 & الأولى ل & المنهج \\
\hline$r v, r$ & 1. & الثانية & التكاملي/الاجتماعي- \\
\hline 促, & rt & الثالثة الثة & الثقافي \\
\hline$r q, \uparrow$ & 1. & الرابعة & \\
\hline iv & 11 & 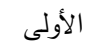 & المهارات المدرسية \\
\hline$\varepsilon r, V$ & 1. & الثانية & الأساسية/يوجهها \\
\hline$r_{0}, .0$ & r & الثالثة & المعلم \\
\hline r T,T & 1. & الرابعة & \\
\hline$r \cdot, 0 T$ & 11 & 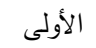 & التعلم المتمركز حول \\
\hline$r v, \varepsilon 0$ & 1. & الثانية & الطفل \\
\hline rr, १^ & rt & 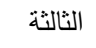 & \\
\hline 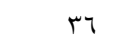 & 1. & الرابعة & \\
\hline iv, vo & 11 & 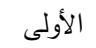 & الدرجة الكلية \\
\hline$\varepsilon r, r_{O}$ & 1. & الثانية & \\
\hline Mr,VV & rt & الثالثة & \\
\hline$r 4, V$ & 1. & الرابعة & \\
\hline
\end{tabular}

للإجابة عن السؤال الثالث الذي نص على "هل توجد فروق في معتقدات الطالبات الهعلمات

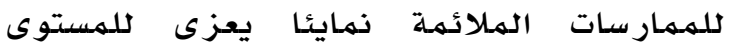
الدراسيء"، تم استخدام تحليل كرو سكال-و اليس اليس

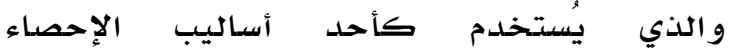

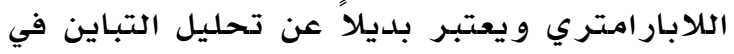

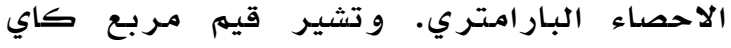

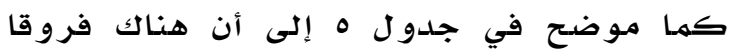
دالة احصائية بين السنوات الأربع في كل من المن

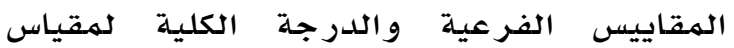

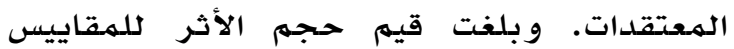
الفرعية و الدرجة الكلية

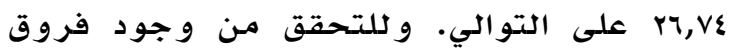

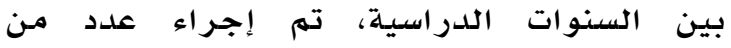

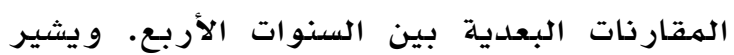
جدول I إلى وجود فروق بين بعض السنوات

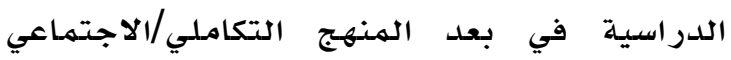
حيث وجد فرق دال بين السنة الأولى والثانية

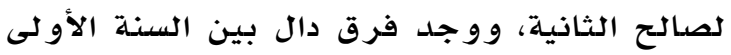

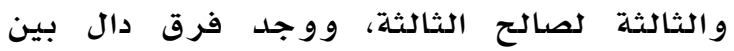
السنة الأولى والررابعة لصالح الرابعة. و وبلغت

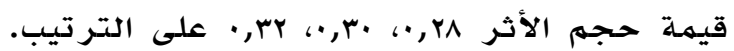

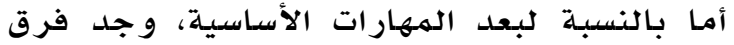

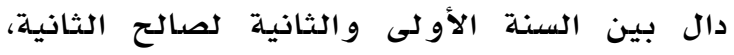

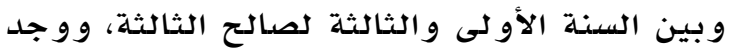
فرق دال بين السنة الأولى والر ابعة لصالح الر ابعة. و بلغت قيمة حجم الأثر بع, •، •r, •، •r, • على الترتيب. بالنسبـة لبعد التعلهم الهتهـركز حول الطفل، وجد فرق دال بين السنة الأولى و الثانية لصالح الثانية، ووجد فرق دال بين السنـة الأولى والثالثة لصالح الثالثة، ووجد فرولد فرق دال 


\section{جدول}

يوضح الفروق بين السنوات الأربع في المقاييس الفرعية واللارجة الكلية لمقياس معتقات المعلم باستخدام اختبار كروكسال-والاس

\begin{tabular}{|c|c|c|c|c|}
\hline الدرجة الكلية & التعلم المتمركز حول الطفل & المهارات المدرسية الأساسية/يوجهه المعلم & المنهج التكاملي/الاجتماعي_التقافي & \\
\hline $10, \mathrm{VA}$ & $\wedge, q$. & IV,rT & $1 \varepsilon, \cdot \varepsilon$ & مربع كا \\
\hline$r,$. & $r, .$. & $r, .$. & $r, .$. & درجات \\
\hline$\cdot, \ldots 1$ & $\cdot, \cdot r_{1}$ & $\cdot, \cdots 1$ & $\cdot, \cdots r$ & مستوى \\
\hline
\end{tabular}

\section{جدول 1 المقائ}

المقارنات البعدية للسنوات المختلفة على المقاييس الفرعية والدرجة الكلية لمقياس معتقات المعلم

\begin{tabular}{|c|c|c|c|c|c|}
\hline مستوى الدلالة & قيمة مربع كا & متوسط الرتب & العدد & السنة & المقياس \\
\hline \multirow[t]{2}{*}{$\cdot, \cdots 7$} & $V, 70$ & $|1, r|$ & 11 & الأولى & المنهج \\
\hline & & $r_{\bullet}, r_{O}$ & 1. & والثانية & التكاملي/الاجتماعي- \\
\hline \multirow[t]{2}{*}{$\cdot, \cdots \varepsilon$} & 11,09 & $1 \leq, 7 V$ & 11 & 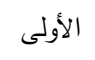 & النقافي \\
\hline & & YO, YV & rt & و الثالثة & \\
\hline \multirow[t]{2}{*}{$\cdot, \ldots r$} & $\Lambda, \vee_{0}$ & $11, \cdot 1$ & 11 & 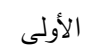 & \\
\hline & & $r \cdot, r_{0}$ & 1. & والرابعة & \\
\hline \multirow{2}{*}{$\cdot, 01 \leqslant$} & $\cdot, \varepsilon r V$ & $1 \wedge, 1$. & 1. & 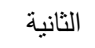 & \\
\hline & & $10, V V$ & rr & والثالثة & \\
\hline \multirow{2}{*}{ 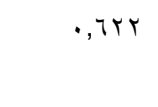 } & $\cdot, r \leq r$ & 9,10 & 1. & الثانية & \\
\hline & & 11,10 & 1. & والرابعة & \\
\hline \multirow[t]{2}{*}{$\cdot, r \leqslant \wedge$} & $\cdot, \wedge \vee q$ & $10, \leqslant 0$ & r & 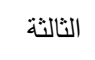 & \\
\hline & & $\curlywedge \wedge, \wedge$. & 1. & والرابعة & \\
\hline \multirow[t]{2}{*}{$\cdot, \cdots 1$} & $|1, r|$ & $|\cdot, 7|$ & 11 & 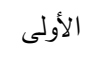 & المهارات المدرسية \\
\hline & & r 1,0 . & 1. & والثانية & الأساسية/يوجهها المعلم \\
\hline \multirow[t]{2}{*}{$\cdot, \cdots 1$} & 11,09 & 15,07 & 11 & 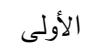 & \\
\hline & & $r 7,1 \wedge$ & rr & و الثالثة & \\
\hline \multirow[t]{2}{*}{$\cdot, \cdot r_{1}$} & $0, r r$ & س & 11 & 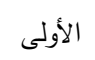 & \\
\hline & & $19, r$. & 1. & والرابعة & \\
\hline \multirow[t]{2}{*}{$\cdot, 10$} & $r, \cdot q$ & $r \cdot, .0$ & 1. & الثانية & \\
\hline & & $1 \leqslant, \wedge 9$ & r & والثالثة & \\
\hline \multirow[t]{2}{*}{$\cdot, Y_{1}$} & $1,0 \mathrm{~V}$ & 1,10 & 1. & 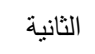 & \\
\hline & & $\wedge, \wedge \circ$ & 1. & والرابعة & \\
\hline \multirow[t]{2}{*}{$\cdot, 779$} & - INT & 17,91 & rt & الثالثة الثة & \\
\hline & & $10, \leqslant 0$ & 1. & و الرابعة & \\
\hline \multirow[t]{2}{*}{$\cdot, .19$} & $0, \Sigma 9$ & $\mid 1, \wedge 1$ & 11 & 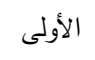 & التعلم المتمركز حول \\
\hline & & $19, r_{0}^{\circ}$ & 1. & والثانية & 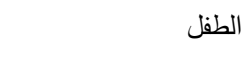 \\
\hline \multirow[t]{2}{*}{$\cdot, \cdot r \leq$} & 0,11 & 10,19 & 11 & 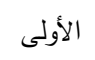 & \\
\hline & & $r \varepsilon, Y V$ & rt & والثالثة & \\
\hline \multirow[t]{2}{*}{ D } & $0, r_{1}$ & $11, \wedge 7$ & 11 & 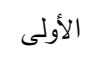 & \\
\hline & & $19, r_{0}$ & 1. & والرابعة & \\
\hline \multirow[t]{2}{*}{$\cdot, \varepsilon \vee 0$} &., 01 & $1 \wedge, r_{0}$ & 1. & 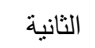 & \\
\hline & & $10, v$. & r & والثالثة & \\
\hline \multirow[t]{2}{*}{$\cdot, \vee \vee$} & $\cdot, \cdot \times 1$ & $1 \cdot, 10$ & 1. & الثانية & \\
\hline & & $1 \cdot, 10$ & 1. & والرابعة & \\
\hline
\end{tabular}


جدول 1 جإن

المقارنات البعدية للسنوات المختلفة على المقاييس الفرعية والدرجة الكلية لمقياس معتقات المعلم

\begin{tabular}{|c|c|c|c|c|c|}
\hline مستوى الدلالة & قيمة مربع كا & منوسط الرتب & العدد & السنة & الكقياس \\
\hline \multirow[t]{2}{*}{$\cdot, 70 \leqslant$} & $\cdot, r \cdot 1$ & 17 & rt & الثالثة & \\
\hline & & $\mid v, 7$ & 1. & والرابعة & \\
\hline \multirow[t]{2}{*}{$\cdot,, \cdots$} & $1 \cdot, \leqslant 9$ & $1 \cdot, 10$ & 11 & الأولى & الدرجة الكلية \\
\hline & & Y, Y, & 1. & والثانية & \\
\hline \multirow[t]{2}{*}{$\cdot, \cdots \varepsilon$} & $\Lambda, r r$ & $1 \leqslant, 7 \varepsilon$ & 11 & الأولى & \\
\hline & & ro,r. & rt & والثالثة & \\
\hline \multirow[t]{2}{*}{$\cdot, \cdots v$} & $V, M T$ & דצr & 11 & الأولى & \\
\hline & & $r \cdot, 10$ & 1. & والرابعة & \\
\hline \multirow[t]{2}{*}{ קTr } & $r, r_{T}$ & $r \cdot, r$. & 1. & الثانية & \\
\hline & & $I \varepsilon, A r$ & rt & والثالثة & \\
\hline \multirow[t]{2}{*}{ TrTH } & $\cdot, 9 \vee 0$ & $11, \wedge$. & 1. & الثانية & \\
\hline & & $q, r$. & 1. & والرابعة & \\
\hline \multirow[t]{2}{*}{$\cdot, \leq 01$} & $\cdot .071$ & 10,74 & rt & الثالثة & \\
\hline & & $1 \wedge, \Gamma_{0}$ & 1. & والرابعة & \\
\hline
\end{tabular}

و اتفقت هذه النتيجة مـع مـا توصل له (Aken, 2013)

الحسالية مـع مـا توصلت له الدراسـة Salellariou) (Smith, 1997) \& Rentzu, 2011) ودراسة (هارون ووشاح، و...r).

وأظهرت النتائج وجود فروق ذات دلالة إحصدائية بين الهشاركات في الدراسة تبعًا لمتغير المعدل

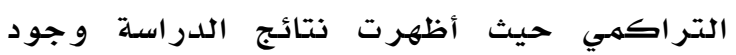
علاقة أرتباطية دالة عند مستوى الدلالة الـئل بين الهعدل التر اكمي للطالبات و الإبعاد الفرعية

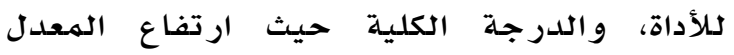

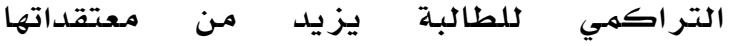

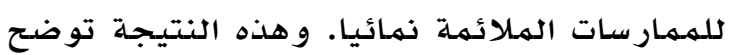

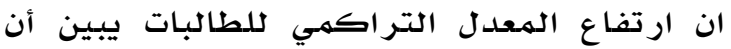

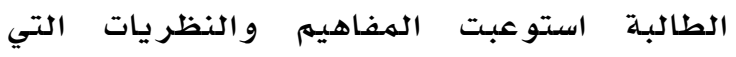
درستها في البر نامه، و تمكنت منها مها انعكس ذلك بصورة إيجابية على معتقداتها للمهمارسات

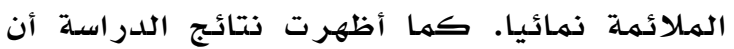
هنالك فروق ذات دلالة إحصائية بين المستجيبات تبعا لمتغير السنوات الدراسية؛ حيث أظهرو دهرت نتائج الدراسـة وجود فروق دالة إحصائيا بين طلبة السنة الأولى والسنوات الثانية و والثالثة و الر ابعة على جميع أبعاد الأداة و لصالح طالبات السنوات الثانية والثالثة والر ابعـة. و هذة النتيجة النة
اجريت هذه الدراسة بهدف الكشف عن درجة

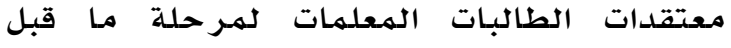
المدررسة، عن المهمارسات الملائمهة نمائيًا

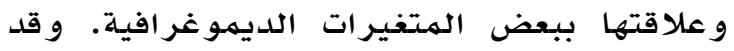
أسفرت الدراسـة عن النتائج التالية:

أظهرت المشاركات في الدراسـة درجة تلفوق المتوسط في مستوى معتقداتهن للمهمارسات الملائمسة نمائيا على جميع فقر ات الاداة و باستثناء عدد قليل منها والمتعلقة بالاختبارات الجمعية، و استخدام الاوراق وكتب الواجبات. و وهذه النتيجة منطقية كون برنامج القسهم مصمهم حسب معايير المسلس القومي لتربية الاطفال

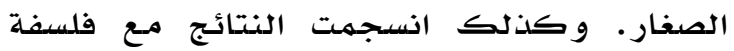

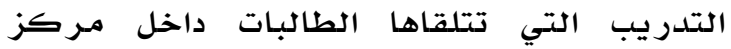
رعاية الطفل التابع للقسم، حيث يقوم المنهاج الهتبع فيه على اسلوب تفريد التعليهم وتصميه خطط فردية تلبي احتياجات كل طفل بشكل

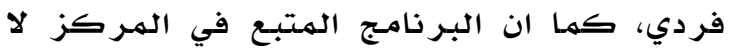
يستخدم الاختبار ات الجمعية في التقييم، وير تكز

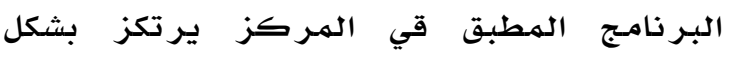

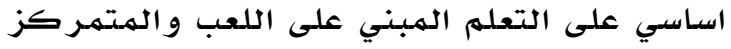
حول الطفل ولا يوجد هناك كتب محلد مادي وواجبات يكلف الاطفال. 


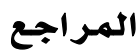

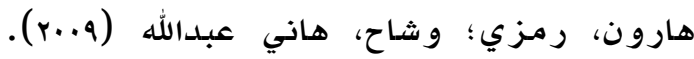

معتقدات معلمات رياض الأطفال في الأردن

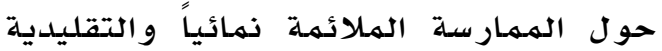

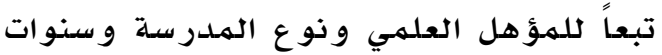

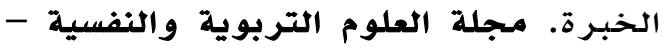

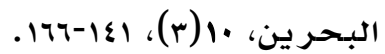

Abbott-Shim, M., Lambert. R. \& McCarty, F. (2003). A comparison of school readiness outcomes for children randomly assigned to a Head Start program and the programs. Journal of Education for Students Placed at Risk, 8(2), 191-214.

Abu-Jaber, M., Al-Shawareb, A., \& Gheith, E. (2010). Kindergarten teachers' beliefs toward developmentally appropriate practice in Jordan. Early Childhood Education Journal. 38. 65-74 EFT ERC.

Bredekamp, S., \& Copple, C. (1997). Developmentally appropriate practice in early childhood programs (pp. 935-989).

Washington. DC: National Association for the Education of Young Children.

Brunning, R., Schraw, G., Norby, M., \& Ronning, R. (2004). Cognitive psychology and instruction. Upper Saddle River. NJ: Merril Prentice Hall.

Buchanan, T., Burts, D., Bidner, J., White, V. \& Charlesworth, R. (1998). Predictors of the developmental appropriateness of the beliefs and practices of first. second and third grade teachers. Early Childhood Research Quarterly. 13, 459-483.

Chaillé, C., \& Silvern, S. (1996). Understanding through play. Childhood Education. 72(5), 274-277.

Charlesworth, R., Hart, C., Burts, D., \& Hernandez, S. (1991). Kindergarten teachers' beliefs and practices. Early Child Development and Care. 70, 17-35.

Charlesworth, R., Hart, C., Burts, D., Mosley, J., \& Fleege, P. (1993). Measuring the developmental appropriateness of kindergarten teachers' beliefs and practices. Early Childhood Research Quarterly. 8,255-276.

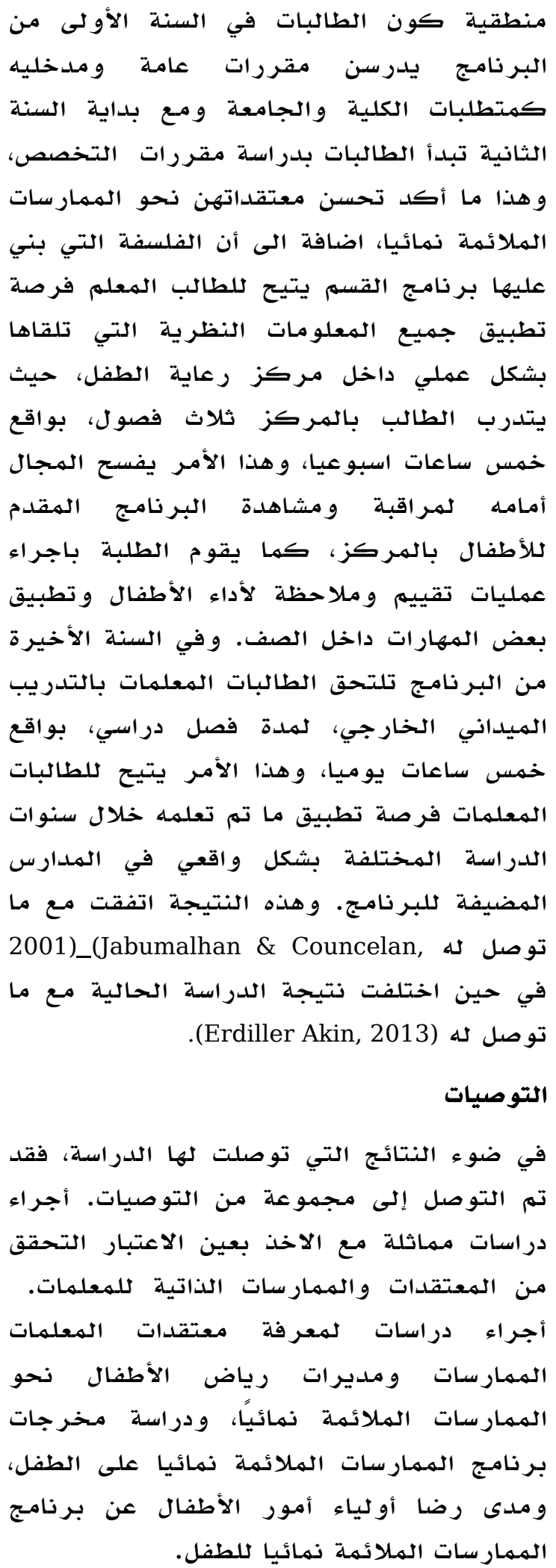


Clark, C., \& Peterson, B. (1986). Teacher's Thought Processes. Handbook of research on teaching. New York: Macmillan.

Cochran-Smith, M., \& Zeichner, K. (2005). Studying Teacher Education: The Report of the AERA Panel on Research and Teacher Education (pp. 816). Mahwah.

Crain, W. (1992). Theories of Development $5^{\text {th }}$ Ed. Pearson Education.

Dodge, D., Colker, L., \& Heroman, C. (2002). The creative curriculum for preschool (4th Ed.). Washington. DC: Teaching Strategies.

Erdiller Akın., Z. (2013). Examining the beliefs of turkish preserves early childhood teachers regarding early childhood curriculum. Journal of Research in Childhood Education. 27, 3, 302-318.

Fang, Z. (1996). A review of research on teacher beliefs and practices. Educational research. 38, 47-65.

File, N., \& Gullo, D. (2002). A comparison of early childhood and elementary education students' beliefs about primary classroom teaching practices. Early Childhood Research Quarterly. 17, 126-137.

Haupt, J., Larsen, J., Robinson, C., \& Hart, C. (1995). The impact of DAP in-service training on the beliefs and practices of kindergarten teachers. Journal of Early Childhood Teacher Education. 16, 12-18.

Jambunathan, S., \& Counselman, K. (2001). Relationship between developmentally appropriate beliefs and practices of preservice teachers. Journal of Early Childhood Teacher Education. 22, 103-108.

Kagan, D. (1992). Implication of research on teacher belief. Educational psychologist. 27, 65-90.

Kim, H. (2011). Developmentally appropriate practice (DAP) as defined and interpreted by early childhood preserves' teachers: Beliefs about DAP and influences of teacher education and field experience. Journal of the Southeastern Regional Association of Teacher Educators. 20(2), 1222.
McMullen, M. (1997). The effects of early childhood academic and professional experience on self perceptions and beliefs about developmentally appropriate practices. Journal of Early Childhood Teacher Education. 18(3). 55-68.

McMullen, M. (1999). Characteristics of teachers who talk the DAP talk and walk the DAP walk. Journal of Research in Childhood Education. 13, 216-230.

Pajares, M. (1992). Teachers' beliefs and educational research: Cleaning up a messy construct. Review of educational research. 62, 307-332.

Rentzou, K., \& Sakellariou, M. (2011a). Greek pre-service kindergarten teachers' beliefs about and practices of developmentally appropriate practices in early childhood education. Early Child Development and Care. 181, 1047-1061.

Rentzou, K., \& Sakellariou, M. (2011b). Greek pre-service kindergarten teachers' beliefs about and practices of developmentally appropriate practices in early childhood education. Early Child Development and Care. 181, 1047-1061.

Sakellariou, M., \& Rentzou, K. (2012a). Comparing beliefs about and practices of developmentally appropriate practices among Greek and Cypriot pre-service kindergarten teachers. Early Child Development and Care. 182 , 1309-1324.

Sakellariou, M., \& Rentzou, K. (2012b). Comparing beliefs and intentions about the importance of teacher-child interactions among Greek and Cypriot pre-service kindergarten teachers. European Early Childhood Education Research Journal. 20 , 233-247.

Sakellariou, M., \& Rentzou, K. (2011a). Cypriot pre-service kindergarten teachers' beliefs about and practices of developmentally appropriate practices in early childhood education. Early Child Development and Care. 181, 1381-1396.

Singer, D. \& Revenson, T. (1997). A Piaget primer: How a child thinks. Madison. CT: International Universities Press. Inc. 
Smith, K. (1997). Student teachers' beliefs about developmentally appropriate practice: Pattern: Stability and the influence of locus of control. Early Childhood Research Quarterly, 12, 221-243.

Stipek, D., \& Byler. P. (1997). Early childhood education teachers: Do they practice what they preach? Early Childhood Research Quarterly. 12, 305-325. 